\title{
An rpw8 quadruple mutant of Arabidopsis Col-0 is partially compromised in bacterial and fungal resistance
}

\author{
Baptiste Castel ${ }^{1}$, Yan $\mathrm{Wu}^{2}$, Shunyuan $\mathrm{Xiao}^{2}$, Jonathan D G Jones ${ }^{1}$ \\ 1 The Sainsbury Laboratory, University of East Anglia, Norwich Research Park, NR4 7UH Norwich, United \\ Kingdom \\ 2 Institute for Bioscience and Biotechnology Research \& Department of Plant Sciences and Landscape \\ Architecture, University of Maryland College Park, Rockville, MD 20850, USA
}

Correspondence should be addressed to SX (xiao@ibbr.umd.edu) or JDGJ (jonathan.jones@tsl.ac.uk)

\begin{abstract}
The plant immune system relies on both cellsurface and intracellular NLR (nucleotidebinding, leucine-rich repeat) receptors. NLRs respond to pathogen effectors and activate effector-triggered immunity: a cocktail of responses, often accompanied by cell death, resulting in resistance.
\end{abstract}

RPW8 encodes an unusual non-NLR Resistance (R) protein and confers broadspectrum powdery mildew resistance. It requires genetic components also required by some NLRs, resembles the HeLo-containing protein MLKL (necroptosis executor in animals) and HET-S (cell death executor in fungi) and is targeted to the extra-haustorial membrane during powdery mildew infection by its $\mathrm{N}$ terminal non-cleaved signal anchor domain. RPW8 displays extensive recent duplication events in Arabidopsis and certain alleles can induce oligomerisation-dependent activation of the NLR RPP7.

All these features enabled us to formulate hypotheses for RPW8 function: (1) RPW8 could be a cell death executor for defence against pathogens. (2) RPW8 could be a decoy for effector targets.

To test these hypotheses, we generated a quadruple knock-out mutant of the four RPW8homologous copies in Arabidopsis Col-0, using CRISPR. The mutant still displays cell death upon activation of four well-characterised NLRs. However, it is partially impaired in powdery mildew resistance and also in bacterial resistance. Interestingly Col-0_rpw8 is delayed in flowering transition. In conclusion, RPW8 plays a broad role in immunity and plant development, beyond resistance to powdery mildew. There is no evidence that it is involved in executing $\mathrm{ETI}$-associated cell death.

\section{Introduction}

Plants have co-evolved with their pathogens for millions of years, driving natural selection for effective immunity. The plant immune system relies on recognition of conserved pathogen-, microbe- or damage-associated molecular patterns (PAMPs, MAMPs and DAMPs) by pattern recognition receptors (PRRs) generally localised at the cell surface. PRR activation leads to $\mathrm{Ca}^{2+}$ influx, mitogen activated protein kinase (MAPK) activation, reactive oxygen species (ROS) production and transcriptional reprogramming, resulting in PAMP-triggered immunity (PTI) (Couto \& Zipfel, 2016). Pathogens evolved effectors to colonize plants. Some effectors interfere with $\mathrm{PTI}$, resulting in effector-triggered susceptibility (ETS). In turn, plants evolved Resistance- $(R$-) genes that recognise effectors. $R$-gene products (i.e. Rproteins) are mainly intracellular. Their activation results in effector-triggered immunity (ETI) which is generally accompanied by salicylic acid production and the hypersensitive response (HR, not to be confused with HR1, $H R 2, H R 3$ and HR4, which are "Homologues of RPW8" Arabidopsis genes), a form of programmed cell death at the site of infection (Jones \& Dangl, 2006). Elevation of salicylic acid levels induces local defence and systemic acquired resistance (Durner et al., 1997). Localized HR is thought to stop propagation of the pathogen within the host (Morel \& Dangl, 1997).

Investigation of the plant immune system revealed hundreds of $R$-genes in most angiosperm genomes. Most of them belong to the Nucleotide-Binding (NB), Apaf-1, R-protein and CED-4 (ARC) and Leucine-rich repeat (LRR) (NLR) family (Kourelis \& Van Der Hoorn, 2018). Earlier this year, cryo-electron 
microscopy was employed to resolve the structure of ZAR1, a plant NLR, in its non-active and active form (Dangl \& Jones, 2019). The non-active form binds ADP in its NB domain and is monomeric (Wang et al., 2019b). The active form binds ATP in its NB domain and is pentameric, forming a resistosome (Wang et al., 2019a) that imposes induced proximity on its $\mathrm{N}$-terminal region. The function of the resistosome is to activate immunity, but the mode of action is not known.

Some components genetically required for NLR function have been characterised. For instance, EDS1 (Enhanced Disease Susceptibility 1) and PAD4 (Phytoalexin Dependent 4) are lipaselike proteins, conserved between many plants and required for TIR-NLR-mediated immunity (Wiermer et al., 2005). TIR-NLRs form a subfamily of plant NLRs that is defined by the presence of an N-terminal TIR (Toll-like, Interleukin-1 receptor and R-protein) domain and specific motifs in the NB-ARC domain. EDS1 is also required redundantly with ICS1 (Isochorismate Synthase 1, required for salicylic acid biosynthesis) for some CC-NLRs (Venugopal et al., 2009). CC-NLRs form a second sub-family of plant NLRs. They are defined by the presence of an N-terminal CC (coiled-coil) domain and specific motifs in the NB-ARC domain.

RPW8 (Resistance to Powdery Mildew 8) is a genetic locus identified in Arabidopsis that confers broad-spectrum resistance to powdery mildew fungi in accession Ms-0. Two homologous R-genes, RPW8.1 and RPW8.2, from the same locus contribute to resistance (Xiao et al., 2001). Transgene analysis showed that adequate expression of RPW8.1 and $R P W 8.2$ also confers resistance to a virulent strain of the oomycete Hyaloperonospora arabidopsidis $(\mathrm{Hpa})$, the cause of downy mildew disease (Wang et al., 2007). Transgenic tobacco plants expressing RPW8.1 and RPW8.2 showed enhanced resistance to powdery mildew (Xiao et al., 2007). More recently, heterologous expression of RPW8. 1 in rice and RPW8.2 in grapevine has also been shown to increase resistance to Magnaporthe oryzae (Li et al., 2018) and powdery mildew (Hu et al., 2018), respectively. Both RPW8.1 and RPW8.2 (noted as RPW8 in later text, unless indicated otherwise) encode a small protein ( 20 kDa) with a predicted CC domain. Intriguingly, RPW8 shares sequence homology to the N-termini of a third sub-family of plant
NLRs, called RPW8-NLRs. This sub-family of NLRs often have only a few members but exists in almost all plants (Zhong \& Cheng, 2016). At least in angiosperms, these RPW8-NLRs are helper NLRs required for signalling of multiple sensor NLRs (Peart et al., 2005; Bonardi et al., 2011; Dong et al., 2016; Qi et al., 2018; Adachi et al., 2019; Castel et al., 2019a; Wu et al., 2019).

Genetic analysis showed that the resistance function of RPW8 requires previously characterised immune components, including EDS1, PAD4 and the salicylic acid pathway, but does not require NDR1 (which is usually required for CC-NLRs), nor COI1 and EIN2 (which regulate jasmonic acid and ethylene signalling respectively) (Xiao et al., 2005). Because EDS1, PAD4 and salicylic acid are generally associated with TIR-NLR-mediated immunity, the above results imply a functional link between RPW8 and TIR-NLR signalling.

During their co-evolution with hosts, some filamentous pathogens, such as powdery mildew and rust fungi, and oomycetes have evolved a common invasive strategy: the formation of haustoria as their feeding structures. Recent studies have shown that the haustorium is encased by a host-derived special membrane called the extra-haustorial membrane (EHM). Interestingly, RPW8.2 is specifically targeted to the EHM during powdery mildew infection (Wang et al., 2009, 2013). The $\mathrm{N}$-terminal domain of RPW8.2, along with two EHM-targeting motifs, is predicted to associate with membranes, and is required for EHM targeting and resistance function of RPW8.2. Intriguingly, the N-terminal portion ( 90 amino acids) of RPW8 shares similarity with MLKL from animals and HELLP and other HeLodomain-containing fungal proteins (Daskalov et al., 2016). MLKL can oligomerise to form pores at the membrane during necroptosis in animals (Murphy et al., 2013). HELLP has a similar function in fungi and can form prions via its $\mathrm{C}$ terminus. Prionisation is associated with membrane targeting and disruption, followed by cell death. A glycine zipper motif conserved between MLKL, HELLP and RPW8 is required for HELLP membrane targeting (Daskalov et al., 2016). In parallel, RPW8 is capable of inducing HR (Xiao et al., 2001) and overexpression of RPW8 can lead to cell death (Xiao et al., 2003, 2005). However, it is not known whether RPW8's membrane-targeting 
and cell death induction share the same molecular basis with that of HELLP and MLKL.

Another interesting feature of the RPW8 locus is the intraspecific gene amplification and diversity within Arabidopsis. RenSeq was conducted on 65 Arabidopsis accessions and revealed two types of sequence variation at the RPW8 locus (Barragan et al., 2019; Van de Weyer et al., 2019). Firstly, there have been a large number of recent duplication events. There are seven sub-clades of $R P W 8$ paralogs: RPW8.1, RPW8.2, RPW8.3, HR1, HR2, HR3 and HR4. Each Arabidopsis accession contains a unique combination of these alleles with different copy numbers. For instance, Ms-0 contains five RPW8 paralogs: RPW8.1 and $R P W 8.2$, which confer resistance to powdery mildew, and $H R 1, H R 2$ and $H R 3$, which are not required for powdery mildew resistance (Xiao et al., 2001). Col-0, which is susceptible to powdery mildew, contains $H R 1, H R 2, H R 3$, and $H R 4$, an additional homolog most closely related to RPW8.1 (Xiao et al., 2001, 2004; Berkey et al., 2017). Interestingly, TueWa1-2 contains as many as 13 RPW 8 paralogs (including three copies of RPW8.1, two of RPW8.2, four of RPW8.3, one of HR1, one of $H R 2$, one of HR3 and one of HR4). The selective advantage of such extreme variation in duplication events is not understood. Secondly, some RPW8 genes encode proteins with intragenic C-terminal duplications. For instance, RPW8.1 from some accessions contains one or more duplications of a 21amino acid motif (Orgil et al., 2007), and HR4 from Col-0 contains five duplications of a 14amino acid motif (Xiao et al., 2004), whereas HR4 from ICE106 contains only two, and HR4 from Fei-0 only one such motif (Barragan et al., 2019).

A large-scale genetic analysis on intraspecific hybrid necrosis revealed that some RPW8 alleles are "incompatible" with alleles of an NLR-type $R$-gene at the RPP7-locus (Chae et al., 2014). Some Arabidopsis crosses display spontaneous necrosis in $F 1$, due to the presence of two incompatible alleles, one coming from each parent. The "Dangerous Mix" $(D M)$ loci have been mapped. Particularly, DM6 (RPP7) and DM7 (RPW8) are responsible for incompatibility in three crosses: Mrk-0 x KZ10, Lerik1-3 x Fei-0 and ICE79 x Don-0. In KZ10 and Fei-0, the RPW8 genes responsible for the phenotype are RPW8.1 and $H R 4$ respectively (Barragan et al., 2019). RPW8.1 ${ }^{\mathrm{KZ} 10}$ has three
C-terminal intragenic repeats, the maximum observed among known RPW8.1 alleles. Interestingly, Arabidopsis accessions carrying an RPW8.1 allele with three C-terminal repeats all form a dangerous mix with Mrk-0. In contrast, HR4Fei-0 has only one C-terminal repeat. Arabidopsis accessions carrying an HR4 allele with one $\mathrm{C}$-terminal repeat all form a dangerous mix with Lerik1-3. The number of $\mathrm{C}$-terminal repeats thus correlates with incompatibility with different RPP7 alleles. However, the function of the repeat is yet to be discovered. In addition, $\mathrm{HR} 4{ }^{\mathrm{Fe}}{ }^{\mathrm{O}-0}$ induces oligomerisation of RPP7b $\mathrm{b}^{\text {Lerik1- }}$ ${ }^{3}$, resulting in $\mathrm{HR}$ in Nicotiana benthamiana ( $\mathrm{Li}$ et al., 2019).

We can formulate two hypotheses about RPW8 function. (1) Based on sequence similarity, RPW8 could function as the HeLo domaincontaining proteins MLKL, HET-S and HELLP from animals and fungi. This function would be a ligand-dependent oligomerization resulting in membrane targeting and disruption for programmed cell death. (2) RPW8 could also be a decoy of effector targets. NRG1 and ADR1 are low copy number and are helper NLRs (Jubic et al., 2019). Their conserved RPW8 domain could in theory provide a potent effector target to suppress immunity mediated by multiple sensor NLRs. RPW8 paralogs could encode decoys for such effectors. They could either trap the effectors to prevent their virulence function or be modified and guarded by NLRs such as RPP7. The extreme degree of recent duplication of RPW8 in Arabidopsis is consistent with this hypothesis.

To test both hypotheses, we generated a quadruple rpw8 mutant (hr1-hr2-hr3-hr4, we called Col-0_rpw8) in Arabidopsis Col-0 to test for possible altered immunity. Our results indicate a function for RPW8 beyond defence against powdery mildew pathogens.

\section{Materials and Methods}

\section{$\underline{\text { Plant genotypes and growth conditions }}$}

Arabidopsis thaliana (Arabidopsis) accessions used in this study is Columbia-0 (Col-0). Col0_eds 1 is an eds1a-eds $1 b$ double CRISPR mutant, published as eds 1-12 (Ordon et al., 2017). Seeds were sown directly on compost and plants were grown at $21^{\circ} \mathrm{C}$, with 10 hours of light and 14 hours of dark, $75 \%$ humidity. For seed collection, 5-week old plants were 
transferred under long-day condition: $21^{\circ} \mathrm{C}$, with 16 hours of light and 8 hours of dark, $75 \%$ humidity. For Nicotiana benthamiana, seeds were sown directly on compost and plants were grown at $21^{\circ} \mathrm{C}$, with cycles of 16 hours of light and 8 hours of dark, $55 \%$ humidity. $N$. benthamiana_nrg1 is an $n r g 1$ CRISPR mutant of $N$. benthamiana (Castel et al., 2019a).

Generation of an hr1-hr2-hr3-hr4 (aka rpw8) null mutant in Arabidopsis Col-0 using CRISPR

CRISPR modules were assembled using the Golden Gate cloning method. The detailed Golden Gate method and protocols can be found in (Engler et al., 2009, 2014; Weber et al., 2011). Specific details for CRISPR module assembly can be found in (Castel et al., 2019b). All the vector used can be found on Addgene (addgene.org). 12 sgRNAs targeting HR1 (TGGCGTCGTGAAGGAGTTGG[nGG], CGACGCCATCATAAGAGCCA[nGG] and GTTCATCGACTTCTTCGGTG[nGG]), HR2 (TGCTCTCCAAATCCTTCACG[nGG], GTCTCGATTCTACAATCTTG[nGG] and GGTTCTTGTCGAAGCTTATG[nGG]), HR3 (GGTTAGTGAGATTATGGCAG[nGG], GTCTTGATGCTACAATCTTT[nGG] and CGATAAGCTTAGCGAAGAAG[nGG]) and HR4 (GCTTGCTGTAATCAAAACAG[nGG], TGGAAAGTATCAGTCCGGTG[nGG] and GGAGACGCGTAAGACTTTCG[nGG]) were designed and assembled by PCR to a sgRNA backbone and 67 bp of the AtU6-26 terminator. sgRNA1 to sgRNA12 were assembled with the AtU6-26 promoter in the Golden Gate compatible level 1 plCH47761, plCH47772, plCH47781, plCH47791, plCH47732, plCH47742, p/CH47751, plCH47761, plCH47772, plCH47781, plCH47791 and plCH47732 respectively. A level 1 vector plCH47811 (with expression in reverse orientation compared to the other level 1 modules) containing a human codon optimized allele of Cas9 under the control of the AtRPS5a promoter and the Pisum sativum rbcS E9 terminator (Addgene: 117505) was assembled with a FAST-Red selectable marker (Addgene: 117499) into a level $M$ vector $p A G M 8031$, using the end-linker p/CH50892. sgRNA1 to sgRNA5 were assembled into a level $M$ vector pAGM8067, using the end-linker p/CH50872. sgRNA7 to sgRNA9 were assembled into a level $M$ vector $p A G M 8043$, using the end-linker pICH50914. sgRNA10 to sgRNA12 were assembled into a level $\mathrm{M}$ vector pAGM8043, using the dummy module p/CH54033 and the end-linker p/CH50881. The four level $M$ modules were assembled into level $P$ vector pICSL4723-P1, using the end-linker plCH79264. Level 1 vectors were cloned using Bsal enzyme and carbenicillin resistance. Level $M$ vectors were cloned using Bpil enzyme and Spectinomycin resistance. Level $P$ vector was cloned using Bsal enzyme and kanamycin resistance. The final vector map can be found in the Supplemental Information. It was expressed via Agrobacterium tumefaciens strain GV3101 in Arabidopsis Col-0. In the first generation after transformation, we recovered somatic mutants. The non-transgenic progeny of a somatic mutant contained a homozygous quadruple knock-out line. One mutation is a 6579 bp deletion between $\mathrm{C} 173$ of $H R 1$ and G22 of HR3. The second mutation is c.28delA in $H R 4$, causing an early stop codon at the $\mathrm{N}$ terminal encoding region. The resulting line lacks functional $H R 1, H R 2, H R 3, H R 4$ and is TDNA free. We called this line Col-0_rpw8.

\section{$\underline{\text { Transcript level measurement }}$}

For gene expression analysis, RNA was isolated from three biological replicates and used for subsequent reverse transcription quantitative PCR (RT-qPCR) analysis. RNA was extracted using the RNeasy Plant Mini Kit (QIAgen) and treated with RNase-Free DNase Set (QIAGEN). Reverse transcription was carried out using the SuperScript IV Reverse Transcriptase (ThermoFisher). qPCR was performed using CFX96 Touch $^{\text {TM }}$ Real-Time PCR Detection System. Primers for qPCR analysis of $P R 1$ are ATACACTCTGGTGGGCCTTACG and TACACCTCACTTTGGCACATCC. Primers for qPCR analysis of EF1a are CAGGCTGATTGTGCTGTTCTTA and GTTGTATCCGACCTTCTTCAGG. Data were analysed using the double delta $\mathrm{Ct}$ method (Livak \& Schmittgen, 2001).

\section{Cell death assay in Arabidopsis}

Pseudomonas fluorescens engineered with a Type Three Secretion system (Pf0-1 EtHAn) (Thomas et al., 2009) carrying pBS46:AvrRps4, pBS46:AvrRps4 KRVY, pBS46:AvrRpt2, pVSP61:AvrRpm1 or pVSP61:AvrPphB were 
grown on selective KB-medium agar plate for 48 hours at $28^{\circ} \mathrm{C}$. Bacteria were harvested from plate, re-suspended in infiltration buffer (10 mM MgCl $2, \mathrm{pH} 5.6$ ) and concentration was adjusted to $\mathrm{OD}_{600}=0.2\left(\sim 10^{8} \mathrm{cfu} / \mathrm{ml}\right)$. The abaxial surface of 4-week old Arabidopsis leaves were hand-infiltrated with $1 \mathrm{ml}$ needleless syringe. Cell death was monitored 24 hours after infiltration.

\section{Cell death assay in N. benthamiana}

A. tumefaciens strains were streaked on selective media and incubated at $28^{\circ} \mathrm{C}$ for 24 hours. A single colony was transferred to liquid LB medium with appropriate antibiotic and incubated at $28^{\circ} \mathrm{C}$ for 24 hours in a shaking incubator (200 rotations per minute). The resulting culture was centrifuged at 3000 rotations per minute for 5 minutes and resuspended in infiltration buffer $(10 \mathrm{mM}$ $\mathrm{MgCl} 2,10 \mathrm{mM}$ MES, $\mathrm{pH} 5.6$ ) at $\mathrm{OD}_{600}=0.4$ $\left(2 \times 10^{8} \mathrm{cfu} / \mathrm{ml}\right)$. For co-expression, each bacterial suspension was adjusted to $\mathrm{OD}_{600}=$ 0.4 in the final mix. The abaxial surface of 4weeks old $N$. benthamiana were infiltrated with $1 \mathrm{ml}$ needle-less syringe. Cell death was monitored three days after infiltration.

Vector used are: RRS1-R ${ }^{\mathrm{K} 1221 \mathrm{Q}-\mathrm{FLAG}}$ and RPS4-HF (Sarris et al., 2015), EDS1-V5 and SAG101-Myc (Huh et al., 2017), NRG1B-HF (Castel et al., 2019a) and HR2-mNeon (this article). HR2 was amplified from Col-0 (Fw: GGCTTAAUATGCCTCTTACCGAGATTATCG

AACCCGAUTTCAAAACGAAGCGAATTC)

and mNeon c-terminal tag was amplified from pICSL50015 (Addgene: 50318) (Fw: ATCGGGTUTGGTGAGCAAGGGAGAGGAG

$\mathrm{G}$,

GGTTTAAUTTACTTGTAAAGCTCGTCCA)

using "KAPA HiFi HotStart Uracil+ ReadyMix (2X)" enzyme (KAPABIOSYSTEMS), following the manufacturer protocol. Amplicons were assembled in a USER compatible vector LBJJ234-OD (containing a FAST-Red selectable marker and a $35 \mathrm{~S}$ / Ocs expression cassette, pre-linearized with $\mathrm{Pacl}$ and $\mathrm{Nt}$. Bbvcl restriction enzymes), with the USER enzyme (NEB), following the manufacturer protocol. The final vector map can be found in the Supplemental Information.

\section{Bacterial growth measurement}

Pseudomonas syringae pv. tomato strain DC3000 carrying pVSP61:AvrRps4-HA or pVSP61 empty vector were grown on selective KB-medium agar plate for 48 hours at $28^{\circ} \mathrm{C}$. Bacteria were harvested from plate, resuspended in infiltration buffer $(10 \mathrm{mM} \mathrm{MgCl}$, $\mathrm{pH}$ 5.6) and concentration was adjusted to $\mathrm{OD}_{600}=0.0005\left(\sim 2.5 \times 10^{5} \mathrm{cfu} / \mathrm{ml}\right)$. The abaxial surface of 5 -week old Arabidopsis leaves were hand-infiltrated with $1 \mathrm{ml}$ needle-less syringe. Plants were covered with a lid for the first 12 hours of bacterial growth. For quantification, leaf samples were harvested with a $6 \mathrm{~mm}$ diameter cork-borer, resulting in a $\sim 0.283 \mathrm{~cm}^{2}$ sized leaf disc. Two leaf discs per leaf were harvested and used as single sample. For each condition, four samples were collected just after infiltration and six samples were collected 72 hours after infiltration. Samples were ground in $200 \mu \mathrm{l}$ of infiltration buffer, serially diluted $(5,50$, 500,5000 and 50000 times) and spotted (5 to $10 \mu \mathrm{l}$ per spot) on selective KB-medium agar plate to grow 48 hours at $28^{\circ} \mathrm{C}$. The number of colonies (cfu per drop) was monitored and the bacterial growth was expressed in $\mathrm{cfu} / \mathrm{cm}^{2}$ of leaf tissue.

\section{Albugo candida propagation}

For propagation of Albugo candida race $2 \mathrm{~V}$ (Rimmer et al., 2000), zoospores were suspended in water $\left(\sim 10^{5}\right.$ spores $\left./ \mathrm{ml}\right)$ and incubated on ice for $30 \mathrm{~min}$. The spore suspension was then sprayed on plants using a Humbrol® spray gun $(\sim 700 \mu \mathrm{l} /$ plant $)$ and plants were incubated at $4{ }^{\circ} \mathrm{C}$ in the dark overnight. Infected plants were kept under 10 hours light $\left(20{ }^{\circ} \mathrm{C}\right)$ and 14 hours dark $\left(16{ }^{\circ} \mathrm{C}\right)$ cycles. Phenotypes were monitored 12 days after spraying.

\section{Powdery mildew propagation}

For testing with powdery mildew, plants were grown under short-day (8 hr light, $16 \mathrm{hr}$ dark) and $75 \%$ relative humidity at $22{ }^{\circ} \mathrm{C}$ for 7 weeks before inoculation with an adapted powdery mildew isolate Golovinomyces cichoracearum $(G c)$ UCSC1 or a non-adapted isolate GC UMSG1 as previously reported (Xiao et al., 2005; Wen et al., 2011). Disease phenotypes with Gc UCSC1 were visually scored and 
photographed. Disease susceptibility was further assessed by counting total number of spores per $\mathrm{mg}$ leaf tissue. At least 6 infected leaves were weighed and combined as one leaf sample, and 4 samples were collected from 12 infected plants for disease quantification. A spore suspension of each sample was made by vortexing the leaves for $1 \mathrm{~min}$ in $10 \mathrm{ml}$ of $\mathrm{H}_{2} \mathrm{O}$ containing $0.02 \%$ Silwet L-77 and used for spore counting using LunaTM Automated Cell Counter (Logos biosysems). Spore counts were normalized to the fresh weight of the leaf samples. For infection tests with Gc UMSG1, which can largely penetrate the cell wall of Arabidopsis but fails to establish micro-colony capable of sporulation (Wen et al., 2011), inoculated leaves were collected at $5 \mathrm{dpi}$ and subjected to trypan blue staining, and total the hyphal length was measured as previously reported (Wen et al., 2011). All infection trials were repeated three times with similar results.

Phylogenetic reconstruction of RPW8 homologs in Arabidopsis Col-0

RPW8 domain boundaries were predicted using SMART (http://smart.emblheidelberg.de/) (Letunic \& Bork, 2017). Amino acid sequences of RPW8 domains were aligned using the MUSCLE method. The evolutionary history was inferred using the Neighbor-Joining method. The evolutionary distances were computed using the Poisson correction method. All positions with less than 95\% site coverage were eliminated. Evolutionary analyses were conducted using the software MEGA7.

\section{Results}

CRISPR mutagenesis enables recovery of an rpw8 quadruple mutant

In Col-0, the four RPW8 homologs HR1, HR2, $H R 3$ and $H R 4$ are located in an $11.8 \mathrm{~kb}$ cluster on Chromosome 3 (Figure 1). To generate null alleles, we designed three sgRNAs per gene, targeting their $\mathrm{N}$-terminal encoding region. Cas9 activity at the sgRNA target could result in large deletion of several genes and/or indels causing early stop codons in individual genes. We expressed the 12 sgRNAs along with Cas9 from a single T-DNA in Arabidopsis Col-0. We identified a line lacking functional $H R 1, H R 2$, $H R 3, H R 4$ that is T-DNA free (see Materials and Methods for more details). We called this line Col-0_rpw8.

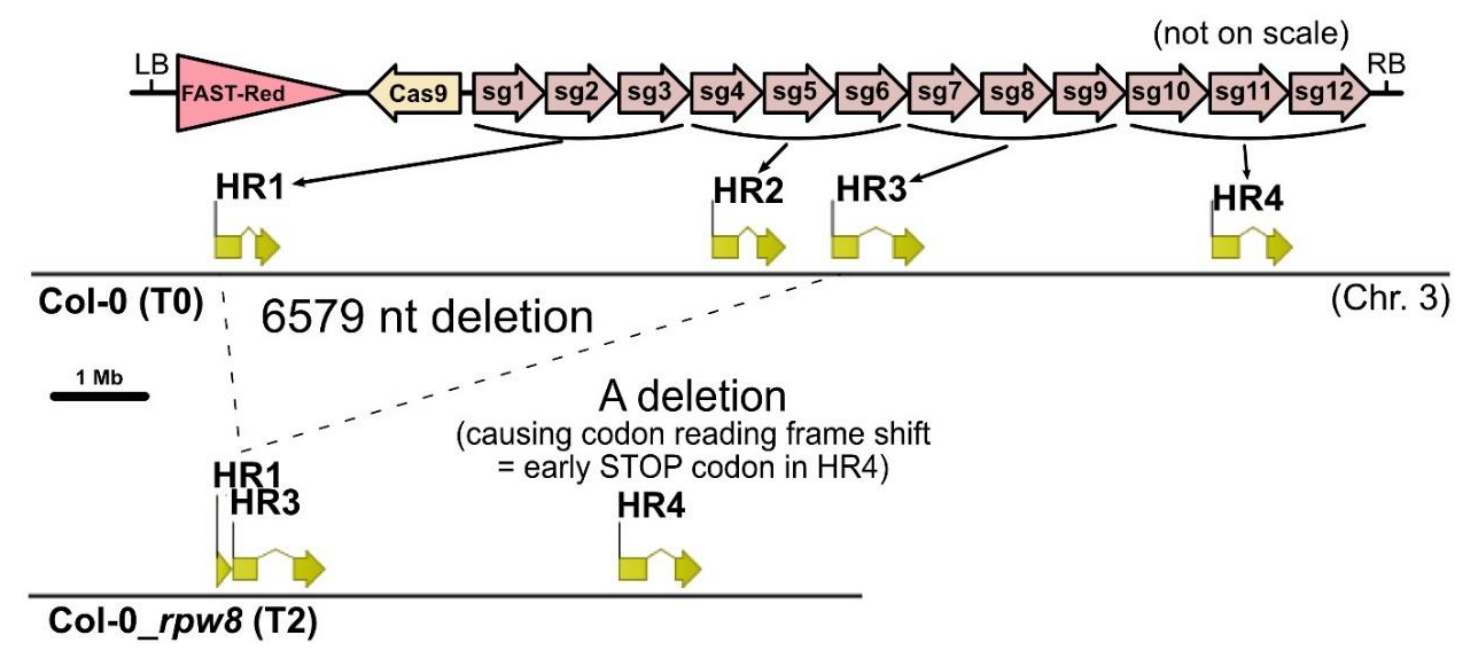

Figure 1: Generating an hr1-hr2-hr3-hr4 (rpw8) null mutant in Arabidopsis Col-0 using
CRISPR

Cas9 (expressed using the RPS5a promoter and E9 terminator) was assembled with 12 sgRNAs (under the U6-26 promoter and terminators) and the FAST-Red selectable marker using the Golden Gate cloning method. Expression of the construct in Col-0 WT resulted in a 6579 bp deletion between $\mathrm{C} 173$ of HR1 and G22 of $H R 3$ and a c.28delA in HR4, causing an early stop codon at the N-terminal encoding region. LB, RB: T-DNA left and right borders. sg: sgRNA. T0: wild type Col-0. T2: Second generation after transformation. Chr. 3: chromosome 3. Construct map is not on scale. RPW8 locus cartoons are on scale. 


\section{rpw8 is slightly autoimmune}

Under normal growth conditions, Col-0_rpw8 plants appear slightly smaller than WT plants (Figure 2A). Mutations that result in activation of constitutive defence often display a dwarf phenotype (van Wersch et al., 2016). The "autoimmunity" is usually associated with elevated salicylic acid (SA) levels, resulting in elevated expression of the SA marker gene $P R 1$. We measured PR1 expression in Col$0 \_$rpw8 to test for autoimmunity. PR1 is
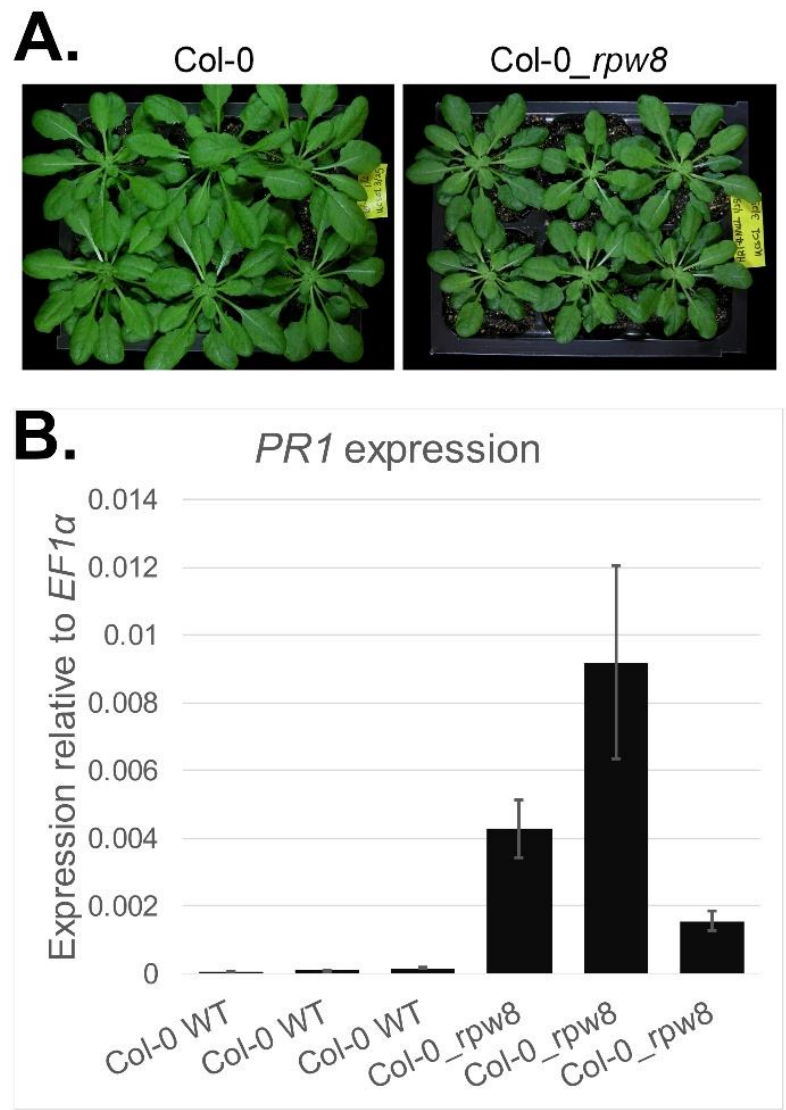

Intriguingly, we found that after growing in short-day ( $8 \mathrm{hr}$ light, $16 \mathrm{hr}$ dark) conditions for 13 weeks, Col-0 plants started bolting, but there was no sign of bolting in Col-0_rpw8 plants (Figure 3). However, there was no noticeable difference among plants of these two significantly more highly expressed in three independent Col-0_rpw8 lines than in WT (Figure 2B). However, it is only expressed at $\sim 0.6 \%$ of the level of EF1a. In contrast, activation of the NLR pair RRS1/RPS4 induces PR1 to 12x the level of EF1a (Castel et al., 2019a). Autoimmune Arabidopsis mutants expressing the effector hopZ5 show PR1 expression levels of $\sim 3$ to $\sim 260$ time more than EF1a (Jayaraman et al., 2017). Thus, the autoimmunity of Col-0_rpw8 is detectable but very low.

\section{Figure 2: Autoimmunity of Col-0 rpw8 is detectable but very low}

A. Col-0_rpw8 plants are slightly smaller than Col-0 WT. Pictures were taken seven weeks after germination. B. PR1 expression is elevated in three independent Col-0_rpw8 lines. The expression level is low compared to the $P R 1$ expression levels in a strong autoimmune mutant or upon NLR activation of WT plants (Jayaraman et al., 2017; Castel et al., 2019a). Each bar represents an individual plant. Error bars represent standard error from three technical replicates. Each of three individual Col-0_rpw8 replicate is significantly different than each of three Col0 WT replicate (paired two-tailed student test, $p$-value $<0.05)$.

genotypes when they were grown in short-day for 4-6 weeks and then shifted to long-day (16 $\mathrm{hr}$ light and $8 \mathrm{hr}$ dark). This unexpected result implies that RPW8 homologs, or perhaps salicylic acid levels, play a role in promoting flowering under short-day conditions. 

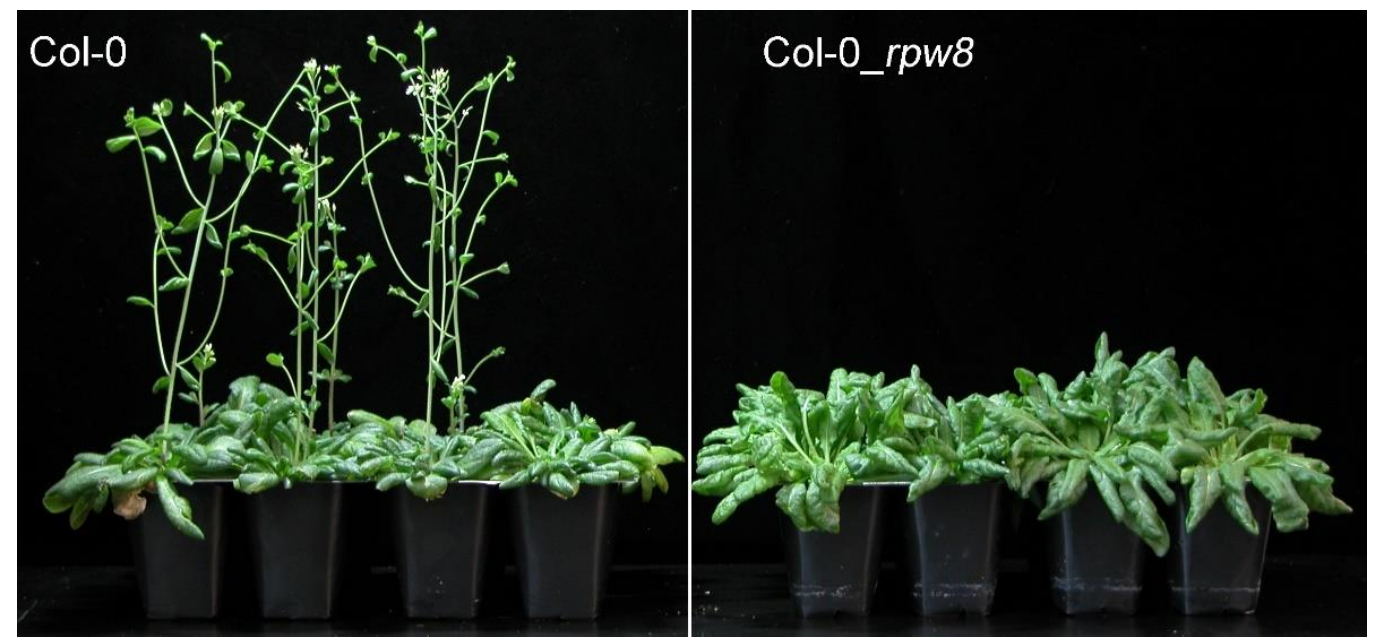

Figure 3: Col-0_rpw8 exhibits later flowering under short-day conditions.

Plants of Col-0 and Col-0_rpw8 were grown under short-day (8 hr light, $16 \mathrm{hr}$ dark) for 13 weeks. While most $(\sim 75 \%)$ of Col-0 plants bolted and the remaining initiated bolting, Col-0_rpw8 plants showed no sign of bolting. Photos were taken at 95 days after seed germination.

RPW8 homologs are not required for cell death mediated by four well-described NLRs

NLR activation often results in a form of cell death called the hypersensitive response (HR). The bacterial effectors AvrRpm1, AvrRpt2, AvrPphB and AvrRps4 can cause an RPM1-, RPS2-, RPS5- and RRS1/RPS4-dependent HR (Kunkel et al., 1993; Grant et al., 1995; Warren et al., 1998; Gassmann et al., 1999) respectively. We delivered these effectors into
Col-0 WT and Col-0_rpw8, using Pf0-1, a nonpathogenic strain of $P$. fluorescens engineered with a Type III Secretion System to deliver an effector of interest into the host cell cytosol (Thomas et al., 2009). AvrRps4 ${ }^{\mathrm{KRVY}}$, an inactive form of AvrRps4, was used as a negative control. Each effector (apart from AvrRps4 KRVY) can still trigger HR in Col-0_rpw8 (Figure 4). These data indicate that RPW8 homologs are not required for RPM1-, RPS2-, RPS5- or RRS1/RPS4-mediated HR.

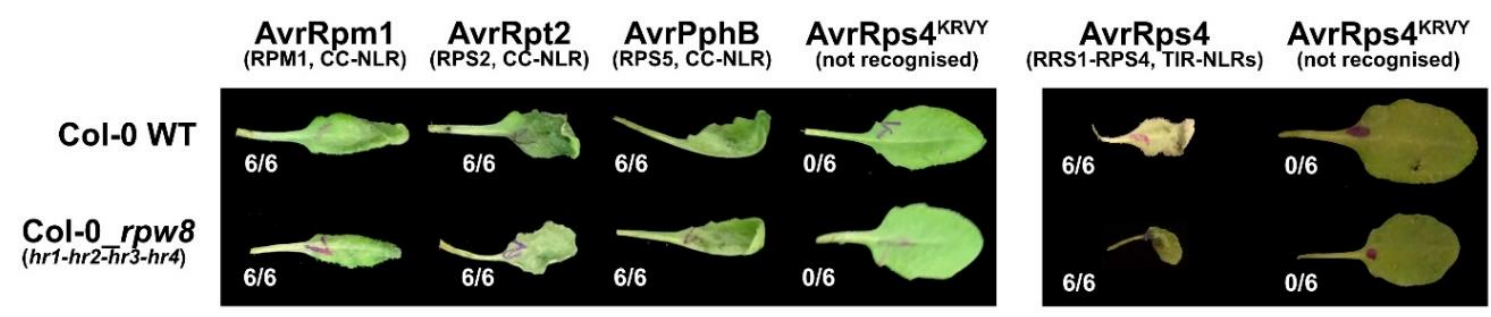

Figure 4: RPW8 homologs are not required for RPM1-, RPS2-, RPS5- or RRS1/RPS4mediated HR

Effectors were delivered using the Pf0-1 system into leaves of 4-week old plants, $\mathrm{OD}_{600}=0.2$, pictures taken $24 \mathrm{hpi}$. Six plants were tested for each combination. Numbers indicate the number of leaves displaying HR. The cognate NLRs (CC- or TIR-) are indicated in parentheses. AvrRps $4^{\mathrm{KRVY}}$ is a non-recognised allele of AvrRps4, used as negative control. AvrRps4 was tested in a different day as the other effectors, hence represented in a separate panel.

We then tested whether RPW8 is sufficient to transduce RRS1/RPS4 signal for HR. EDS1, SAG101 and NRG1 are the three major components of TIR-NLR signalling for HR in Arabidopsis and in Nicotiana benthamiana (Lapin et al., 2019). We transiently expressed 
RRS1-R ${ }^{\mathrm{K} 1221 \mathrm{Q}}$ (an RPS4-dependent auto-active form of RRS1-R) and RPS4 with EDS1 and/or SAG101 and/or NRG1B and/or HR2 from Arabidopsis. The minimal requirement to reconstruct RRS1/RPS4-mediated HR in $N$. benthamiana is EDS1/SAG101/NRG1, but HR2 is dispensable (Figure $\mathbf{5}$ and $\mathbf{S 1}$ ). This indicates that Col-0 RPW8 is neither necessary nor sufficient to transduce RRS1/RPS4 signal for HR. Parenthetically, it also indicates that $N$. benthamiana alleles of SAG101/EDS1/NRG1 cannot transduce the RRS1/RPS4 response, while Arabidopsis alleles can.

\begin{tabular}{|c|c|c|c|c|c|c|c|c|c|}
\hline AtRRS1-R ${ }^{K 1221 Q}-F L A G$ & + & + & + & + & + & + & + & + & + \\
\hline AtRPS4-HF & + & + & + & + & + & + & + & + & + \\
\hline AtEDS1-V5 & & + & & + & & & + & + & + \\
\hline AtSAG101-Myc & & & + & + & & & + & + & + \\
\hline AtNRG1B-HF & & & & & + & & + & & + \\
\hline AtHR2-mNeon & & & & & & + & & + & + \\
\hline
\end{tabular}

Figure 5: RRS1/RPS4-mediated HR in $N$. benthamiana requires EDS1/SAG101/NRG1, but not HR2

RRS1-R ${ }^{\mathrm{K} 1221 \mathrm{Q}}$ (an RPS4-dependent auto-active form of RRS1-R) and RPS4 with EDS1 and/or SAG101 and/or NRG1B and/or HR2 from Arabidopsis, were transiently expressed in N. benthamiana. $A$. tumefaciens strains GV3101 carrying denoted construct were infiltrated in 4-week old leaves, OD $600=$ 0.04. Pictures were taken three days after infiltration. Three pictures per combination indicates infiltration of three different plants. Red indicates full HR. Orange indicates partial HR. Figure S1 displays more control combinations from the same assay.

Col-0 rpw8 mutant supports more growth of a virulent bacterial pathogen

RRS1/RPS4-mediated HR is not RPW8dependent (Figure 4 and Figure 5). Consistently, the growth of $P$. syringae pv tomato strain DC3000 carrying AvrRps4 is not affected in Col-0_rpw8 (Figure 6A and S2A-B). AvrRps 4 avirulence function observed in Col-0, is lost in Col-0_eds1 but maintained in Col- 0_rpw8, indicating that RPW8 is not required for RRS1/RPS4-mediated resistance. In contrast, the growth of DC3000 carrying an empty vector is significantly enhanced in Col-0_rpw8 compared to that in Col-0 (Figure 6B and S2CD). In fact, Col-0_rpw8 is as susceptible as Col0_eds1, indicating that loss of all RPW8 homologs compromises resistance to bacterial pathogens. 

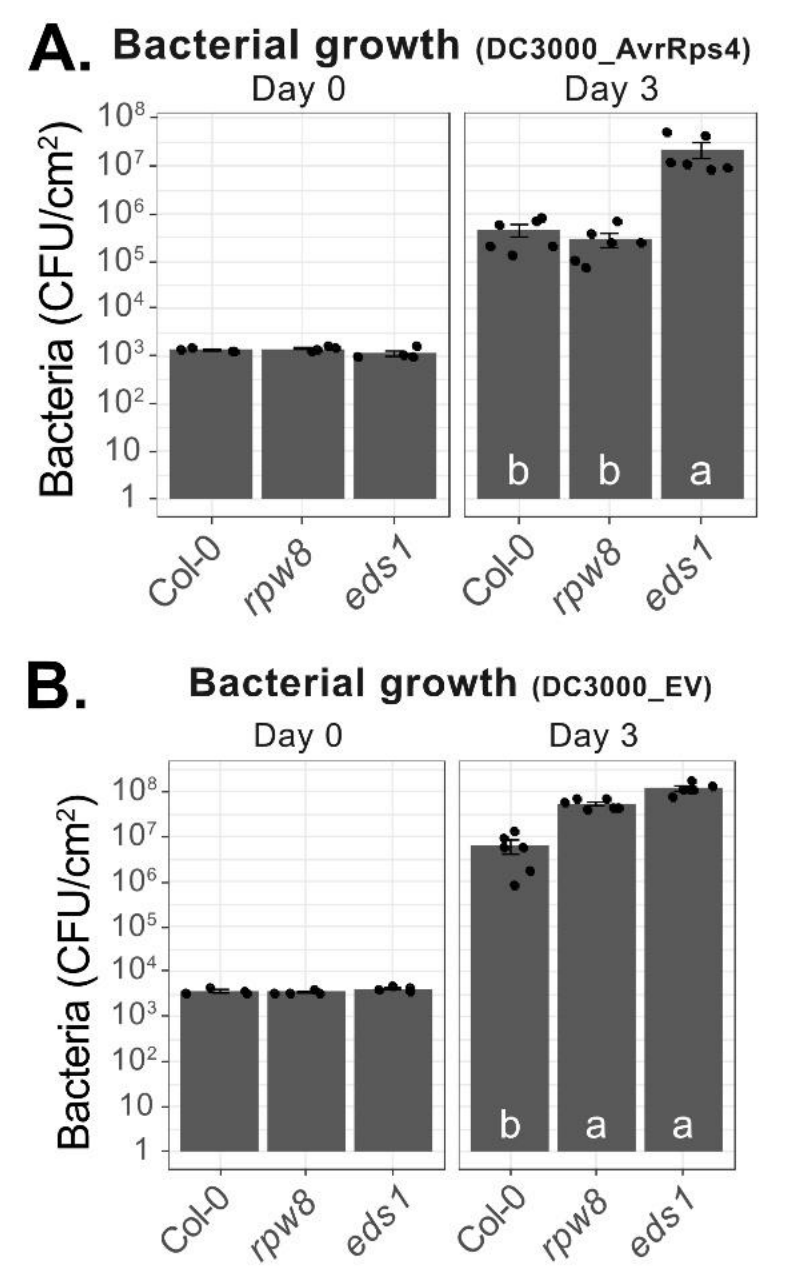

RPW8 homologs are not required for WRR4Amediated resistance to Albugo candida

Albugo candida is an oomycete causing white rust in Brassicaceae. The race Ac2V, isolated from Brassica juncea in Canada, can grow on Arabidopsis transgressive segregants or eds 1 mutants (Rimmer et al., 2000; Cevik et al.,

\section{Figure 7: Ac2V resistance does not require RPW8 in Col-0}

5 -week old plants were sprayed inoculated with Ac2V. Plants were phenotyped 12 days after inoculation. Abaxial and adaxial picture of the same leaf are showed. Numbers indicate the number of individual plants showing similar phenotype out of the number of plants tested. Col-0 WT and Col-0_rpw8 are resistant. Col$0 \_$eds 1 is susceptible.

Col-0 rpw 8 is more susceptible to adapted and non-adapted powdery mildew fungi
Figure 6: Resistance to an avirulent $P$. syringae strain containing AvrRps4 is not affected whereas growth of a virulent $P$. syringae strain is enhanced in Col-0_rpw8.

Five-week old plants were infiltrated with $P$. syringae pv. tomato strain DC3000, carrying AvrRps4 (A) or empty vector $(E V, B)$ at $\mathrm{OD}_{600}=0.0005$. Bacterial quantification was performed just after infiltration (Day 0) and at $3 \mathrm{dpi}$ (Day 3). Each dot represents one individual plant. Letters indicate significant differences $(P<0.05)$ as determined by a one-way ANOVA followed by post hoc Tukey's honestly significant difference (HSD) analysis. CFU $/ \mathrm{cm}^{2}$ : colony-forming unit per square centimetre of leaf. Two more biological replicates are displayed in Figure S2.
2019). Ac2V is resisted by $W R R 4 A, W R R 4 B$ and an unknown recessive or haplo-insufficient $R$-gene in Col-0 (Borhan et al., 2008; Cevik et al., 2019). We tested Col-0, Col-0_rpw8 and Col-0_eds1 with Albugo candida Ac2V and found that resistance conferred by the aforementioned $R$-genes requires $E D S 1$ but not RPW8 (Figure 7).

\section{Albugo candida race $\mathrm{Ac2V}$}

resisted by WRR 4A, WRR 4B and an unknown gene in Col-0

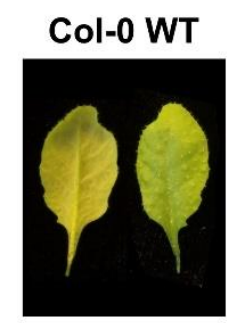

Resistant $(8 / 8)$

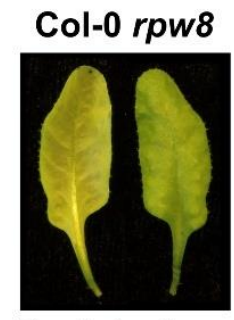

Resistant (8/8)

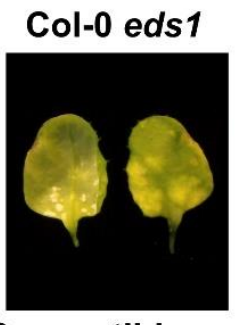

Susceptible (8/8)
Col-0 is susceptible to the adapted (virulent) powdery mildew isolate Gc UCSC1 but still 
mounts SA-dependent basal resistance against it (Xiao et al., 2005). To test if and how much the four RPW8 homologs contribute to the basal resistance, we inoculated eight weeks-old plants of Col-0 and Col-0_rpw8 with GC UCSC1. At $10 \mathrm{dpi}$, Col-0_rpw8 plants support more fungal growth than Col-0 plants based on visual scoring in three independent experiments (one representative is shown in Figure 8A). Quantification of disease susceptibility showed that Col-0_rpw8 plants produced $\sim 1.7 x$ spores per $\mathrm{mg}$ fresh leaf tissue (Figure 8B).
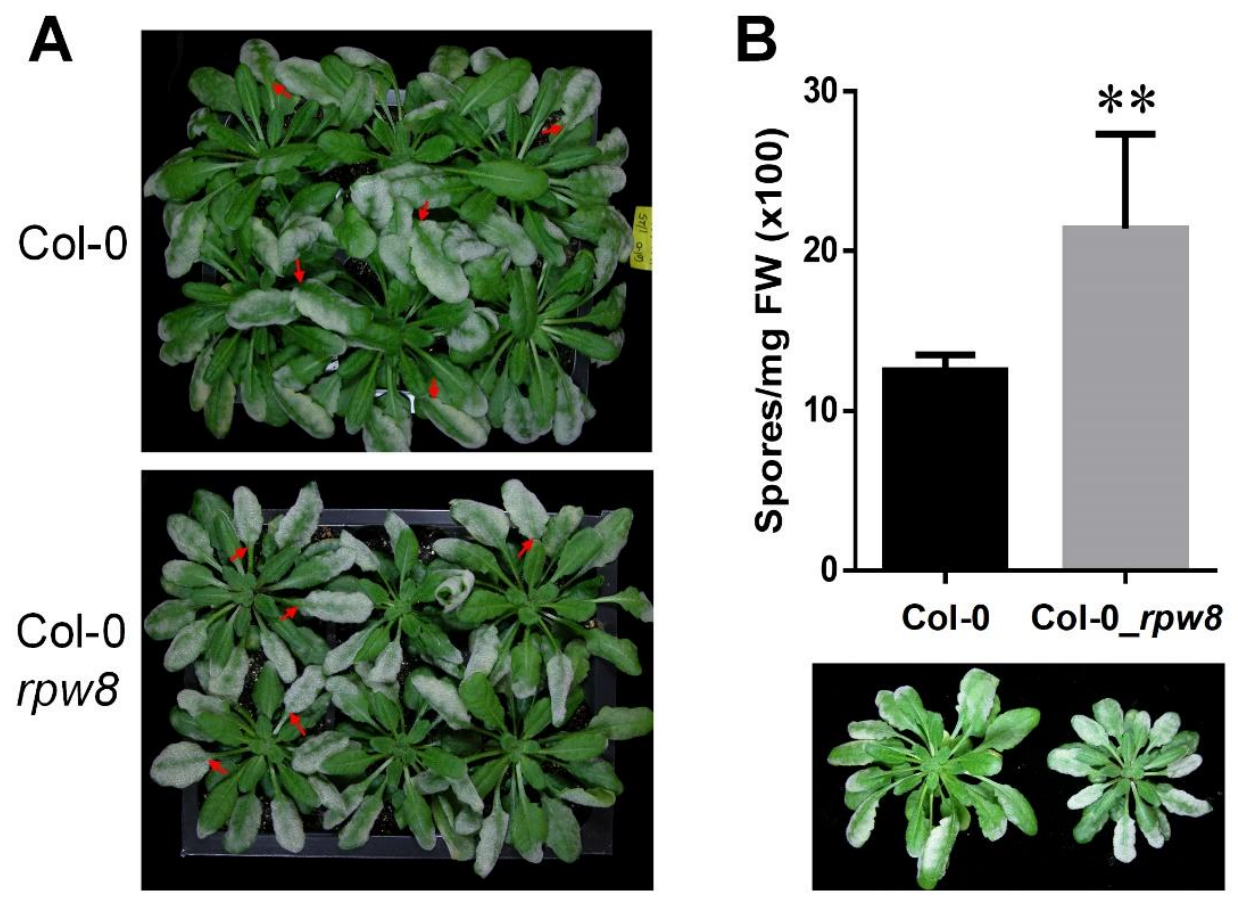

Figure 8: Col-0_rpw8 is more susceptible to an adapted powdery mildew.

(A) Eight week-old plants were inoculated with Gc UCSC1. Pictures were taken at 10 dpi. Note, leaves of Col-0_rpw8 were more evenly covered by whitish mildew compared to those of Col-0 (indicated by red arrows). (B) Representative infected leaves were weighed and subjected to quantification of total spores per mg fresh leaf tissue. ${ }^{* *}$ indicates significant difference (student $t$-test; $\mathrm{P}<0.01$ ).

In contrast, Col-0 and 24 other tested Arabidopsis accessions were completely resistant to Gc UMSG1, which can only develop very short hyphae and then is arrested shortly after spore germination (Wen et al., 2011). To test if RPW8 homologs are involved in resistance against non-adapted powdery mildew, we inoculated plants of Col-0 and Col-
$0 \_r p w 8$ with Gc UMSG1 and measured total hyphal length at 5 dpi. We found that Col$0 \_$rpw8 plants supported much more extensive hyphal growth compared to Col-0 (Figure 9), indicating that RPW8 homologs in Col-0 collectively contribute to basal resistance against non- or poorly-adapted powdery mildew. 

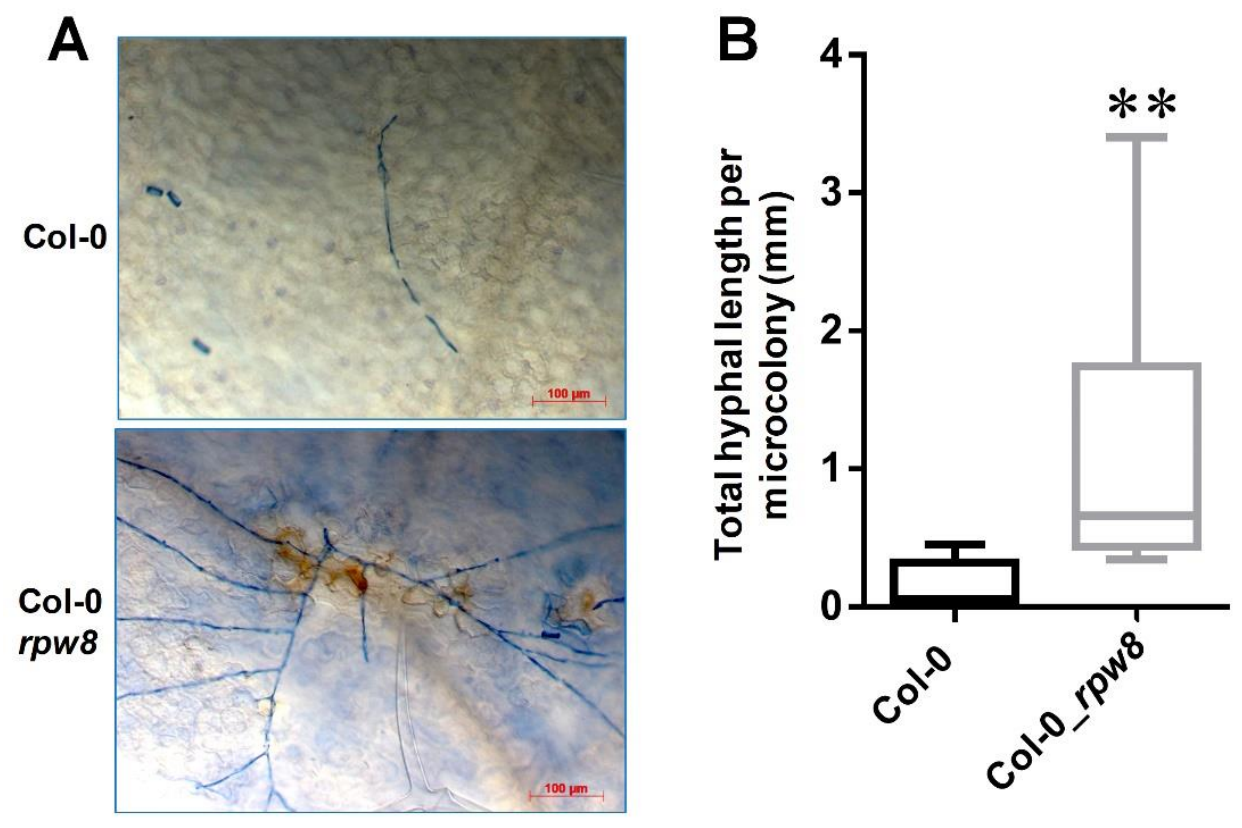

\section{Figure 9: Col-0_rpw8 supports more hyphal growth of a non-adapted powdery mildew.}

Eight weeks-old plants were inoculated with Gc UMSG1. Leaves were subjected to trypan blue staining at $5 \mathrm{dpi}$. (A) Representative microcolony of the indicated genotypes. (B) Total hyphal length of microcolonies. At least 20 microcolonies were measured for total hyphal length for each genotype. ${ }^{* *}$ indicates significant difference (student $t$-test; $\mathrm{P}<0.01$ ).

\section{Discussion}

Based on previous characterisation of RPW8 (Xiao et al., 2005; Collier et al., 2011; Barragan et al., 2019; Li et al., 2019), we propose the following two hypotheses that could explain the molecular and biochemical function of RPW8. The first hypothesis posits that RPW8 homologs and the RPW8 domain in RPW8NLRs participate in activation of immune responses through oligomerization and membrane insertion (pore-forming), thus contributing to HR and pathogen resistance. The recent analysis revealing similarities between RPW8 and the HeLo-domaincontaining proteins MLKL, HELLP and HET-S from animals and fungus (Daskalov et al., 2016) supports this hypothesis. In animals and fungi, these HeLo-domain-containing proteins can oligomerise (upon sensing of various signals) causing the HeLo-domain to form a disruptive membrane insertion structure (i.e. poreforming), resulting in cell death (Cai et al., 2017). Based on the presence of a putative Nterminal HeLo domain, MLKL-encoding genes have recently been identified in plants (Mahdi et al., 2019). Ablation of all the three $M L K L$ genes in Arabidopsis compromised resistance against biotrophic pathogens. These plant MLKL proteins can form tetramers and are associated with microtubules, suggesting a cell deathindependent immunity mechanism (Mahdi et al., 2019). How these MLKL proteins enhance plant immunity remains unresolved. It is possible that RPW8 homologs and plant MLKLlike proteins define a HeLo-domain-containing protein family in plants and serve similar functions as their fungal and animal counterparts. If so, RPW8-NLRs such as ADR1 and NRG1 could then be renamed HeLo-NLRs. The earlier observations that RPW8.2 and other RPW8 homologs are localized to the EHM (Wang et al., 2009; Berkey et al., 2017) and over-expression of RPW8 results in massive cell death (Xiao et al., 2003) support this hypothesis. However, the precise molecular function of the putative N-terminal HeLodomain of MLKL and RPW8 has yet to be characterized.

In this study, we showed that the HR triggered by five well-described NLRs does not require any RPW8 homologs in Arabidopsis (Figure 4). In addition, HR2 is not sufficient to reconstruct 
RRS1/RPS4-triggered HR in N. benthamiana (Figure 5). By contrast, loss of all four RPW8 homologs compromised resistance against $P$. syringae and powdery mildew (Figure 6, Figure 8 and Figure 9). These observations, together with the earlier findings that RPW8 enhances resistance against an oomycete (Hpa) and interacts with RPP7 (Wang et al., 2007; Li et al., 2019), suggest that RPW8 plays an important role in plant immunity but is dispensable for the HR. However, unlike plant MLKLs whose role in cell death is unclear (Mahdi et al., 2019), overexpression of wildtype RPW8 homologs or variants or C-terminal YFP-tagged HR3 results in cell death (Xiao et al., 2003; Wang et al., 2013; Berkey et al., 2017). Since all tested RPW8 homologs are capable of membrane targeting during infection (Wang et al., 2013; Berkey et al., 2017), RPW8 function could be to direct the associated protein complex to a specific subcellular compartment to activate local immune response, including cell death in extreme cases.

According to the second hypothesis, RPW8 proteins are decoys for RPW8-NLR-targeting effectors. However, such avirulent effectors are not known. Even if they exist, the loss of their function in Col-0_rpw8 would not been seen if other avirulent effectors get recognised by an RPW8-independent mechanism. Given that the pathogens tested in our study contain up to several hundreds of effectors, this may well be the case. Thus, our results neither validate or invalidate this RPW8 decoy hypothesis. Characterisation of an RPW8-targeted effector would enable further investigation of this question.

A detailed search for RPW8 and RPW8 domain revealed 11 predicted proteins in Col-0 (Zhong \& Cheng, 2016). Four are Homologues of RPW8 (HR1, HR2, HR3 and HR4), five are RPW8-containing NLRs (ADR1, ADR1-L1, ADR1-L2, NRG1A and NRG1B), one is a noncanonical NLR (DAR5: RPW8-NB-ARC-LIM) and the last one is encoded by AT3G26470. The predicted protein encoded by AT3G26470 has 221 amino acid protein $(25.3 \mathrm{kDa})$ and contains an N-terminal RPW8 domain. AT3G26470 displays a two-exons structure, typical for RPW8. We thus consider this gene a distant Homologue of RPW8 and called it HR5. However, the RPW8 domain of HR5 resembles more the RPW8 domain of ADR1-L1 than that of NRG1 or HR1, HR2, HR3 and HR4 (Figure
S3). Hence, HR5 might have resulted from a recent duplication event of the ADR1-L1 RPW8 domain. Similar to the above, HR5 could be a decoy for an ADR1-L1-targeting effector, independently of any HR1, HR2, HR3 and/or HR4 function. Parsimony suggests that HR1, HR2, HR3 and HR4 share a common ancestor, which is not shared with HR5. Thus, if HR1, HR2, HR3 and HR4 play a redundant function, it is not likely to be shared with HR5.

A recent study highlighted co-evolution between EDS1, SAG101 and NRG1 within plant clades to regulate downstream pathways in TIR-NLR-mediated immunity. Particularly, these three components need to have coevolved to be functional (Lapin et al., 2019). In this study, we tested whether HR2 is part of the minimal required components to reconstruct RRS1/RPS4-triggered HR in N. benthamiana. We found that co-expression of Arabidopsis EDS1, SAG101 and NRG1 is necessary to reconstitute RRS1/RPS4-triggered HR in $N$. benthamiana, but HR2 is dispensable (Fig. 5). Thus, RPW8 is neither required nor sufficient, so likely not involved, in RRS1-RPS4-mediated HR. We confirmed that EDS1, SAG101 and NRG1 need to come from the same genome to function, as previously reported (Lapin et al., 2019). However, unlike Roq1 (a TIR-NLR from $N$. benthamiana) that can signal via EDS1/SAG101/NRG1 from either Arabidopsis or $N$. benthamiana genomes (Lapin et al., 2019), RRS1-RPS4 can only fully signal via Arabidopsis EDS1/SAG101/NRG1. It indicates that the TIR-NLRs Roq1 and RPS4 are sensed differentially by EDS1, SAG101 and NRG1. Recently, TIR domains of plant NLRs have been shown to possess enzymatic activity (Horsefield et al., 2019; Wan et al., 2019). They can degrade nicotinamide adenine dinucleotide in its oxidized form (NAD+) into adenine dinucleotide ribose (ADPR), variant-cyclic ADPR (v-cADPR) and nicotinamide (Nam) (Wan et al., 2019). Our data show that $N$. benthamiana EDS1/SAG101/NRG1 does not sense RRS1/RPS4 activity, but Arabidopsis EDS1/SAG101/NRG1 can, even in the $N$. benthamiana system. This suggests a more complex signal transduction pathway between the activation of TIR-NLRs, enzymatic activity and activation of EDS1/SAG101/NRG1.

In conclusion, we generated an hr1-hr2-hr3-hr4 quadruple mutant (called rpw8 mutant) in Arabidopsis Col-0. NLR-mediated phenotypes tested remain intact in the mutant. However, 
Col-0_rpw8 is partially compromised in resistance against powdery mildew and $P$. syringae, and surprisingly in flowering under short-day growth conditions. Future research is needed to characterize the precise mechanism by which RPW8 proteins or domains contribute to immunity, in comparison to the HeLocontaining proteins in fungi and animals.

\section{Acknowledgments}

Work at The Sainsbury Laboratory (BC and JDGJ) was supported by the Gatsby Foundation (http://www.gatsby.org.uk/). Work at the University of Maryland (YW and SX) was supported by a National Science Foundation grant (IOS-1457033). The authors thanks Mark Youles (Synbio at The Sainsbury Laboratory) for help in plasmid vector development.

\section{References}

Adachi H, Derevnina L, Kamoun S. 2019. NLR singletons, pairs and networks: evolution, assembly and regulation of the intracellular immunoreceptor circuitry of plants. Current Opinion in Plant Biology 50: $121-131$

Barragan CA, Wu R, Kim S, Xi W, Habring A, Hagmann J. 2019. RPW8 / HR Repeats Predict NLRdependent Hybrid Performance. PLoS Genetics 15: e1008313.

Berkey R, Zhang Y, Ma X, King H, Zhang Q, Wang W, Xiao S. 2017. Homologues of the RPW8 Resistance Protein Are Localized to the Extrahaustorial Membrane that Is Likely Synthesized De Novo. Plant Physiology 173: 600-613.

Bonardi V, Tang S, Stallmann A, Roberts M, Cherkis KA, Dangl JL. 2011. Expanded functions for a family of plant intracellular immune receptors beyond specific recognition of pathogen effectors. Proceedings of the National Academy of Sciences 108: 16463-16468.

Borhan MH, Gunn N, Cooper A, Gulden S, Tör M, Rimmer SR, Holub EB. 2008. WRR4 encodes a TIR-NB-LRR protein that confers broad-spectrum white rust resistance in Arabidopsis thaliana to four physiological races of Albugo candida. Molecular Plant-Microbe Interactions 21: 757-768.

Cai X, Xu H, Chen ZJ. 2017. Prion-like polymerization in immunity and inflammation. Cold Spring Harbor Perspectives in Biology 8: a023515.

Castel B, Ngou PM, Cevik V, Kim DS, Yang Y, Ding P, Jones JDG. 2019a. Diverse NLR immune receptors activate defence via the RPW8-NLR NRG1. New Phytologist 222: 966-980.

Castel B, Tomlinson L, Locci F, Yang Y, Jones JDG. 2019b. Optimization of T-DNA architecture for Cas9-mediated mutagenesis in Arabidopsis. PLOS ONE 14: e0204778.

Cevik V, Boutrot F, Apel W, Robert-Seilaniantz A, Furzer O, Redkar A, Castel B, Kover P, Prince D, Holub EB, et al. 2019. Transgressive segregation reveals mechanisms of Arabidopsis immunity to Brassica-infecting races of white rust (Albugo candida). Proceedings of the National Academy of Sciences 116: 2767-2773.

Chae E, Bomblies K, Kim ST, Karelina D, Zaidem M, Ossowski S, Martín-Pizarro C, Laitinen RAE, Rowan BA, Tenenboim H, et al. 2014. Species-wide genetic incompatibility analysis identifies immune genes as hot spots of deleterious epistasis. Cell 159: 1341-1351.

Collier SM, Hamel L-P, Moffett P. 2011. Cell death mediated by the N-terminal domains of a unique and highly conserved class of NB-LRR protein. Molecular Plant-Microbe Interactions 24: 918-931.

Couto D, Zipfel C. 2016. Regulation of pattern recognition receptor signalling in plants. Nature Reviews Immunology 16: 537-552.

Dangl JL, Jones JDG. 2019. A pentangular plant inflammasome. Science 364: 31-32.

Daskalov A, Habenstein B, Sabaté R, Berbon M, Martinez D, Chaignepain S, Coulary-Salin B, Hofmann K, Loquet A, Saupe SJ. 2016. Identification of a novel cell death-inducing domain reveals 
that fungal amyloid-controlled programmed cell death is related to necroptosis. Proceedings of the National Academy of Sciences 113: 2720-2725.

Dong OX, Tong M, Bonardi V, El Kasmi F, Woloshen V, Wünsch LK, Dangl JL, Li X. 2016. TNLmediated immunity in Arabidopsis requires complex regulation of the redundant $A D R 1$ gene family. New Phytologist 210: 960-973.

Durner J, Shah J, Klessig DF. 1997. Salicylic acid and disease resistance in plants. Trends in Plant Science 2: 266-274.

Engler C, Gruetzner R, Kandzia R, Marillonnet S. 2009. Golden gate shuffling: A one-pot DNA shuffling method based on type lls restriction enzymes. PLoS ONE 4: e5553.

Engler C, Youles M, Gruetzner R, Ehnert TM, Werner S, Jones JDG, Patron NJ, Marillonnet S. 2014. A Golden Gate modular cloning toolbox for plants. ACS Synthetic Biology 3: 839-843.

Gassmann W, Hinsch ME, Staskawicz BJ. 1999. The Arabidopsis RPS4 bacterial-resistance gene is a member of the TIR-NBS-LRR family of disease-resistance genes. Plant Journal 20: 265-277.

Grant MR, Godiard L, Straube E, Ashfield T, Lewald J, Sattler A, Innes RW, Dangl JL. 1995. Structure of the Arabidopsis RPM1 Gene Enabling Dual Specificity Disease Resistance. Science 269: 843-846.

Horsefield S, Burdett H, Zhang X, Manik MK, Shi Y, Chen J, Qi T, Gilley J, Lai J-S, Rank MX, et al. 2019. NAD+ cleavage activity by animal and plant TIR domains in cell death pathways. Science 365: 793-799.

Hu Y, Li Y, Hou F, Wan D, Cheng Y, Han Y, Gao Y, Liu J, Guo Y, Xiao S, et al. 2018. Ectopic expression of Arabidopsis broad-spectrum resistance gene RPW8.2 improves the resistance to powdery mildew in grapevine (Vitis vinifera). Plant Science 267: 20-31.

Huh SU, Cevik V, Ding P, Duxbury Z, Ma Y, Tomlinson L, Sarris PF, Jones JDG. 2017. Proteinprotein interactions in the RPS4/RRS1 immune receptor complex. PLOS Pathogens 13: e1006376.

Jayaraman J, Choi S, Prokchorchik M, Choi DS, Spiandore A, Rikkerink EH, Templeton MD, Segonzac C, Sohn KH. 2017. A bacterial acetyltransferase triggers immunity in Arabidopsis thaliana independent of hypersensitive response. Scientific Reports 7: 3557.

Jones JDG, Dangl JL. 2006. The plant immune system. Nature 444: 323-329.

Jubic LM, Saile S, Furzer OJ, El Kasmi F, Dangl JL. 2019. Help wanted: helper NLRs and plant immune responses. Current Opinion in Plant Biology 50: 82-94.

Kourelis J, Van Der Hoorn RAL. 2018. Defended to the Nines: 25 years of Resistance Gene Cloning Identifies Nine Mechanisms for R Protein Function. The Plant Cell 30: 285-299.

Kunkel BN, Bent AF, Dahlbeck D, Innes RW, Staskawicz BJ. 1993. RPS2, an Arabidopsis disease resistance locus specifying recognition of Pseudomonas syringae strains expressing the avirulence gene avrRpt2. The Plant Cell 5: 865-875.

Lapin D, Kovacova V, Sun X, Dongus J, Bhandari DD, Born P von, Bautor J, Guarneri N, Stuttmann J, Beyer A, et al. 2019. A coevolved EDS1-SAG101-NRG1 module mediates cell death signaling by TIR-domain immune receptors. The Plant Cell: 10.1101/572826.

Letunic I, Bork P. 2017. 20 years of the SMART protein domain annotation resource. Nucleic Acids Research 46: D493-D496.

Li L, Habring A, Weigel D. 2019. Oligomerization of the NLR immune receptor RPP7 triggered by the atypical resistance protein RPW8 / HR as a ligand. bioRxiv: 682807.

Li Y, Zhang Y, Wang QX, Wang TT, Cao XL, Zhao ZX, Zhao SL, Xu YJ, Xiao ZY, Li JL, et al. 2018. RESISTANCE TO POWDERY MILDEW8.1 boosts pattern-triggered immunity against multiple pathogens in Arabidopsis and rice. Plant Biotechnology Journal 16: 428-441.

Livak KJ, Schmittgen TD. 2001. Analysis of relative gene expression data using real-time quantitative PCR and the 2- $\triangle \Delta C T$ method. Methods 25: 402-408. 
Mahdi L, Huang M, Zhang X, Nakano T, Brulé Kopp L, Saur IML, Jacob F, Kovacova V, Lapin D, Parker JE, et al. 2019. Plant mixed lineage kinase domain-like proteins limit biotrophic pathogen growth. bioRxiv: 681015.

Morel J-B, Dangl JL. 1997. The hypersensitive response and the induction of cell death in plants. Cell Death and Differentiation 4: 671-683.

Murphy JM, Czabotar PE, Hildebrand JM, Lucet IS, Zhang JG, Alvarez-Diaz S, Lewis R, Lalaoui $\mathbf{N}$, Metcalf D, Webb Al, et al. 2013. The pseudokinase MLKL mediates necroptosis via a molecular switch mechanism. Immunity 39: 443-453.

Ordon J, Gantner J, Kemna J, Schwalgun L, Reschke M, Streubel J, Boch J, Stuttmann J. 2017. Generation of chromosomal deletions in dicotyledonous plants employing a user-friendly genome editing toolkit. Plant Journal 89: 155-168.

Orgil U, Araki H, Tangchaiburana S, Berkey R, Xiao S. 2007. Intraspecific genetic variations, fitness cost and benefit of RPW8, a disease resistance locus in Arabidopsis thaliana. Genetics 176: 2317-2333.

Peart JR, Mestre P, Lu R, Malcuit I, Baulcombe DC. 2005. NRG1, a CC-NB-LRR protein, together with N, a TIR-NB-LRR protein, mediates resistance against tobacco mosaic virus. Current Biology 15: 968-973.

Qi T, Seong K, Thomazella DPT, Kim JR, Pham J, Seo E, Cho M-J, Schultink A, Staskawicz BJ. 2018. NRG1 functions downstream of EDS1 to regulate TIR-NLR-mediated plant immunity in Nicotiana benthamiana. Proceedings of the National Academy of Sciences 115: E10979-E10987.

Rimmer SR, Mathur S, Wu CR. 2000. Virulence of isolates of Albugo candida from western Canada to Brassica species. Canadian Journal of Plant Pathology 22: 229-235.

Sarris PF, Duxbury Z, Huh SU, Ma Y, Segonzac C, Sklenar J, Derbyshire P, Cevik V, Rallapalli G, Saucet SB, et al. 2015. A Plant Immune Receptor Detects Pathogen Effectors that Target WRKY Transcription Factors. Cell 161: 1089-1100.

Thomas WJ, Thireault CA, Kimbrel JA, Chang JH. 2009. Recombineering and stable integration of the Pseudomonas syringae pv. syringae $61 \mathrm{hrp} / \mathrm{hrc}$ cluster into the genome of the soil bacterium Pseudomonas fluorescens Pf0-1. Plant Journal 60: 919-928.

Venugopal SC, Jeong R-D, Mandal MK, Zhu S, Chandra-Shekara AC, Xia Y, Hersh M, Stromberg AJ, Navarre D, Kachroo A, et al. 2009. Enhanced Disease Susceptibility 1 and Salicylic Acid Act Redundantly to Regulate Resistance Gene-Mediated Signaling. PLoS Genetics 5: e1000545.

Wan L, Essuman K, Anderson RG, Sasaki Y, Monteiro F, Chung E-H, Osborne Nishimura E, DiAntonio A, Milbrandt J, Dangl JL, et al. 2019. TIR domains of plant immune receptors are NAD+cleaving enzymes that promote cell death. Science 365: 799-803.

Wang W, Devoto A, Turner JG, Xiao S. 2007. Expression of the membrane-associated resistance protein RPW8 enhances basal defense against biotrophic pathogens. Molecular plant-microbe interactions 20: 966-976.

Wang J, Hu M, Wang J, Qi J, Han Z, Wang G, Qi Y, Wang H-W, Zhou J-M, Chai J. 2019a.

Reconstitution and structure of a plant NLR resistosome conferring immunity. Science 364 : eaav5870.

Wang J, Wang J, Hu M, Qi J, Wang G, Han Z, Qi Y, Wang H-W, Zhou J-M, Chai J. 2019b. Ligandtriggered allosteric ADP release primes a plant NLR complex. Science 364: eaav5868.

Wang W, Wen Y, Berkey R, Xiao S. 2009. Specific Targeting of the Arabidopsis Resistance Protein RPW8.2 to the Interfacial Membrane Encasing the Fungal Haustorium Renders Broad-Spectrum Resistance to Powdery Mildew. The Plant Cell 21: 2898-2913.

Wang W, Zhang Y, Wen Y, Berkey R, Ma X, Pan Z, Bendigeri D, King H, Zhang Q, Xiao S. 2013. A comprehensive mutational analysis of the Arabidopsis resistance protein RPW8.2 reveals key amino acids for defense activation and protein targeting. The Plant cell 25: 4242-4261.

Warren RF, Henk A, Mowery P, Holub EB, Innes RW. 1998. A mutation within the leucine-rich 
repeat domain of the arabidopsis disease resistance gene RPS5 partially suppresses multiple bacterial and downy mildew resistance genes. Plant Cell 10: 1439-1452.

Weber E, Engler C, Gruetzner R, Werner S, Marillonnet S. 2011. A modular cloning system for standardized assembly of multigene constructs. PLOS ONE 6: e16765.

Wen Y, Wang W, Feng J, Luo MC, Tsuda K, Katagiri F, Bauchan G, Xiao S. 2011. Identification and utilization of a sow thistle powdery mildew as a poorly adapted pathogen to dissect post-invasion non-host resistance mechanisms in Arabidopsis. Journal of Experimental Botany 62: 2117-2129.

van Wersch R, Li X, Zhang Y. 2016. Mighty Dwarfs: Arabidopsis Autoimmune Mutants and Their Usages in Genetic Dissection of Plant Immunity. Frontiers in Plant Science 7: 1717.

Van de Weyer A-L, Monteiro F, Furzer OJ, Nishimura MT, Cevik V, Witek K, Jones JDG, Dangl JL, Weigel D, Bemm F. 2019. A Species-Wide Inventory of NLR Genes and Alleles in Arabidopsis thaliana. Cell 178: 1260-1272.e14.

Wiermer M, Feys BJ, Parker JE. 2005. Plant immunity: the EDS1 regulatory node. Current Opinion in Plant Biology 8: 383-389.

Wu Z, Li M, Dong OX, Xia S, Liang W, Bao Y, Wasteneys G, Li X. 2019. Differential regulation of TNL-mediated immune signaling by redundant helper CNLs. New Phytologist 222: 938-953.

Xiao S, Brown S, Patrick E, Brearley C, Turner JG. 2003. Enhanced Transcription of the Arabidopsis Disease Resistance Genes RPW8.1 and RPW8.2 via a Salicylic Acid - Dependent Amplification Circuit Is Required for Hypersensitive Cell Death. The Plant cell 15: 33-45.

Xiao S, Calis O, Patrick E, Zhang G, Charoenwattana P, Muskett P, Parker JE, Turner JG. 2005. The atypical resistance gene, RPW8, recruits components of basal defence for powdery mildew resistance in Arabidopsis. Plant Journal 42: 95-110.

Xiao S, Charoenwattana P, Holcombe L, Turner JG. 2007. The Arabidopsis Genes RPW8.1 and RPW8.2 Confer Induced Resistance to Powdery Mildew Diseases in Tobacco . Molecular PlantMicrobe Interactions 16: 289-294.

Xiao S, Ellwood S, Calis O, Patrick E, Li T, Coleman M, Turner JG. 2001. Broad-spectrum mildew resistance in Arabidopsis thaliana mediated by RPW8. Science 291: 118-120.

Xiao S, Emerson B, Ratanasut K, Patrick E, O'Neill C, Bancroft I, Turner JG. 2004. Origin and maintenance of a broad-spectrum disease resistance locus in Arabidopsis. Molecular Biology and Evolution 21: 1661-1672.

Zhong Y, Cheng Z-M (Max). 2016. A unique RPW8-encoding class of genes that originated in early land plants and evolved through domain fission, fusion, and duplication. Scientific Reports 6: 32923.

\section{Supplemental information}


bioRxiv preprint doi: https://doi.org/10.1101/839308; this version posted November 12,2019 . The copyright holder for this preprint (which was not certified by peer review) is the author/funder, who has granted bioRxiv a license to display the preprint in perpetuity. It is made available under aCC-BY-NC-ND 4.0 International license.

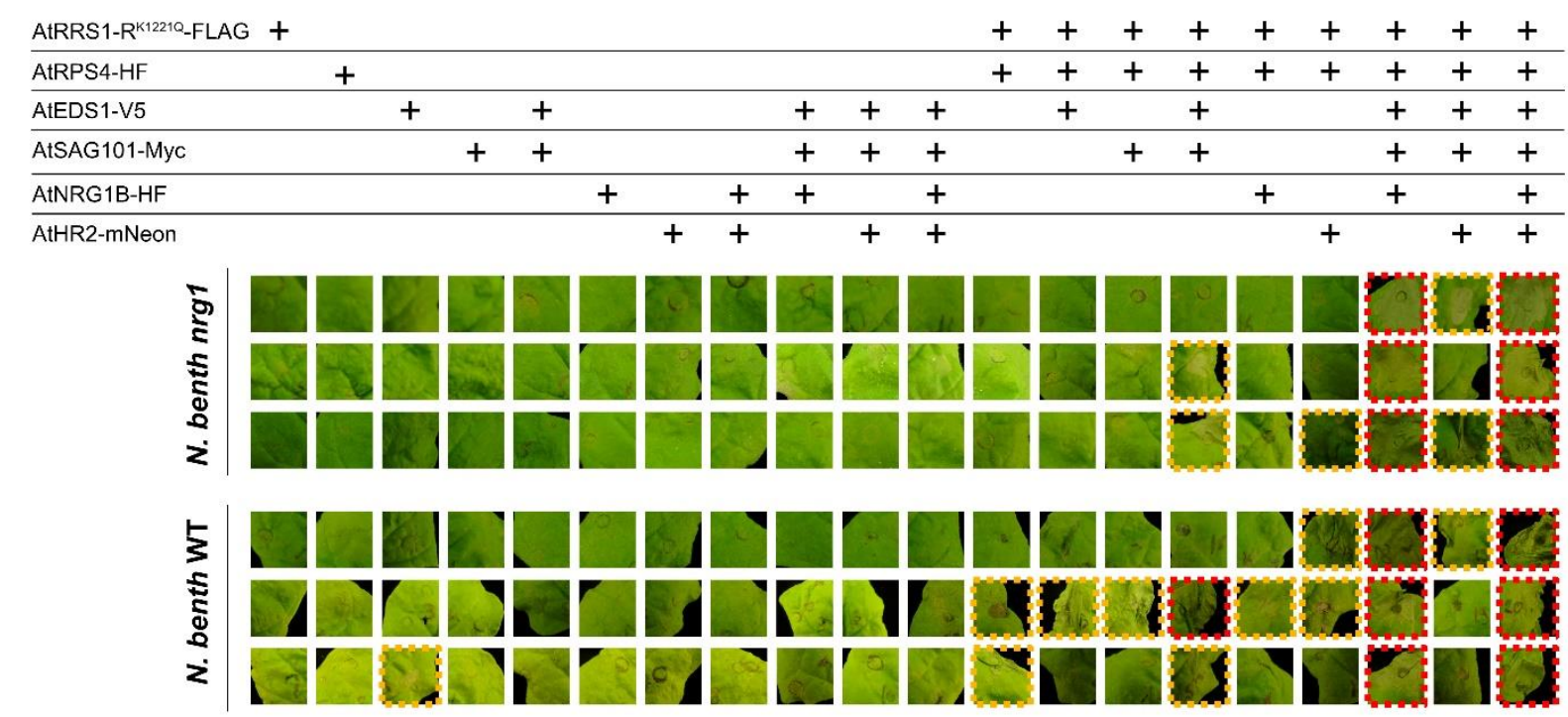

Figure S1: RRS1/RPS4-mediated HR in $N$. benthamiana requires EDS1/SAG101/NRG1, but not HR2

RRS1-R ${ }^{\mathrm{K} 1221 \mathrm{Q}}$ (an RPS4-dependent auto-active form of RRS1-R) and RPS4 with EDS1 and/or SAG101 and/or NRG1B and/or HR2 from Arabidopsis, were transiently expressed in 4-week old N. benthamiana. A. tumefaciens strain GV3101 carrying denoted construct were infiltrated in 4-week old leaves, $\mathrm{OD}_{600}=0.04$. Pictures were taken $3 \mathrm{dpi}$. Three pictures per combination indicates infiltration of three different plants. Red indicates full HR. Orange indicates partial HR. Figure $\mathbf{5}$ is a partial summary of this assay. 
A. Bacterial growth (DC3000_AvrRps4)

Day 0
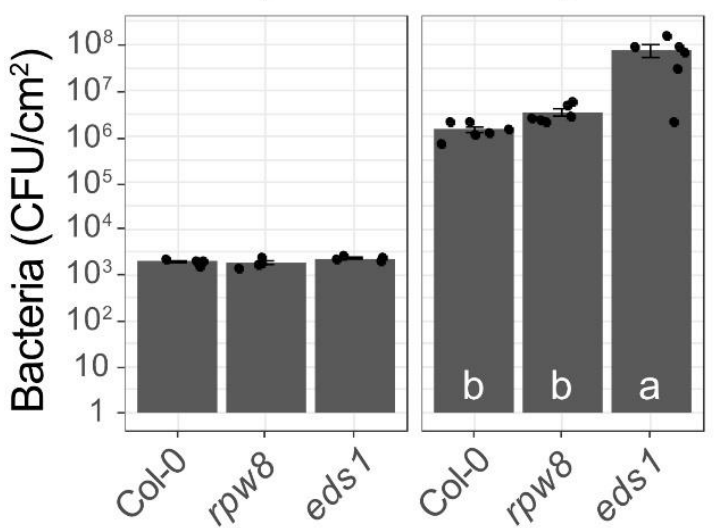

C.

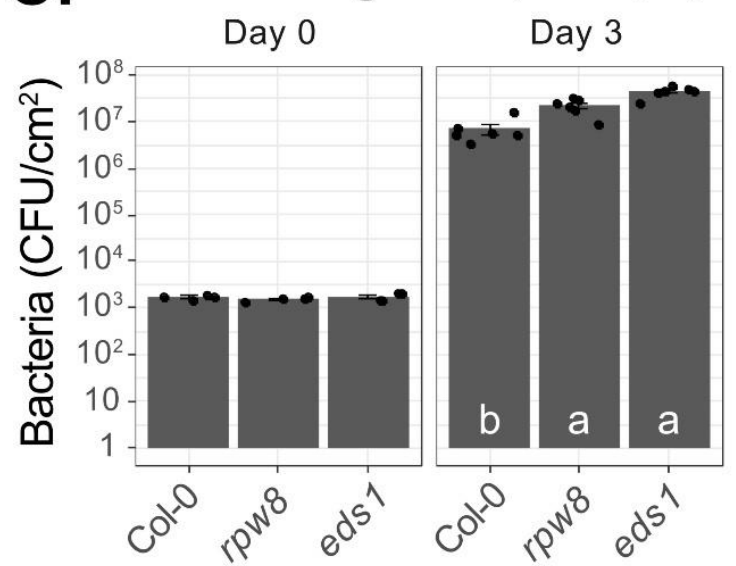

\section{B. Bacterial growth (DC3000_AvrRps4)}

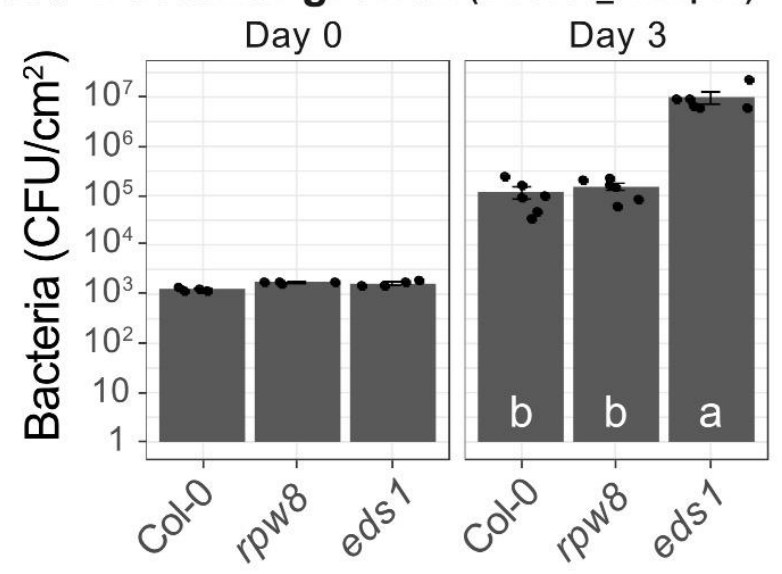

D.

Figure S2: $P$. syringae growth is RPW8-dependent, unlike AvrRps4 avirulence function

Five-week old plants were infiltrated with P. syringae pv. tomato strain DC3000, carrying AvrRps4 (A. and B.) or empty vector (EV, C. and D.) at $\mathrm{OD}_{600}=0.0005$. Bacterial quantification was performed just after infiltration (Day 0 ) and at $3 \mathrm{dpi}$ (Day 3). Each dot represents one individual plant. Each panel represent an independent experiment (plant grown at different time). Letters indicate significant differences $(P<0.05)$ as determined by a one-way ANOVA followed by post hoc Tukey's honestly significant difference (HSD) analysis. CFU/ $\mathrm{cm}^{2}$ : colony-forming unit per square centimetre of leaf. This figure displays biological replicates of Figure 6.

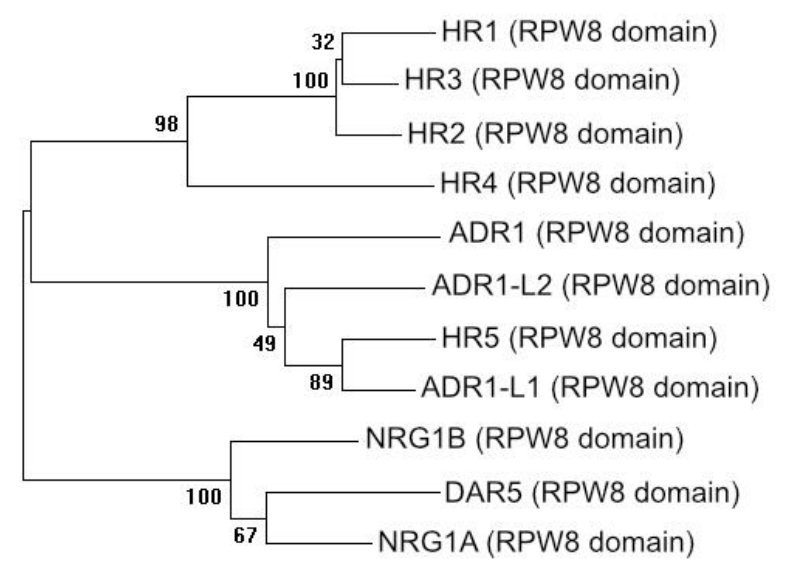

Figure S3: A fifth RPW8 homolog is related to ADR1-L1 RPW8 domain

Neighbor-joining phylogeny of the amino acid sequences of RPW8 domains (identified with SMART, http://smart.embl-heidelberg.de/) from all RPW8containing proteins in Col-0. Number indicates value of bootstrap 100. The tree is drawn to scale, with branch lengths in the same units as those of the evolutionary distances used to infer the phylogenetic tree. Evolutionary distances are expressed in number of amino acid substitutions per site (Poisson correction method). Tree made using the software MEGA7. 